\title{
Ayurvedic management of Thrombo Angiitis Oblitrerans (T. A. O.) (Buerger's disease) - A case study
}

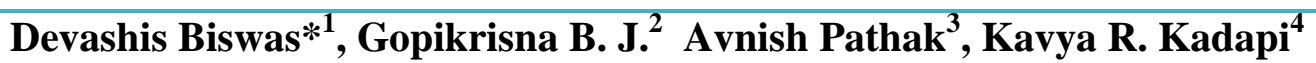

1. P. G. Scholar,

2. Professor and H. O. D.

3. Associate Professor and Pro H.O.D.

4. P. G. Scholar,

Department of Shalyatantra, Sri Dharmasthala Manjunatheswara College of Ayurved and Hospital, Hassan, Karnataka- India.

*Corresponding Author: Email-vaidyadevashisbiswas@gmail.com; Ph: 9686325174

\section{Abstract:}

T.A.O. (Thrombo Angiitis Oblitrerans) also called as buerger's disease or smoker's disease. It is due to in response to the inflatinmation of all three layer of the artery. It is non atherosclerotic inflammatory disorder involving medium sized and distal arteries. Mainly occurs in young males between ages of 20 a prears and is common in lower limbs. The common cause of the disease is smoking or tobacco chewing. Tobacco secretes nicotine which is responsible for vasospas, In early stage it is unilateral but later involves both the limbs. Causes include hypersensitivity to cigarette, recurrent minor feet injuries, poor hygiene and altered autonomic functions. Sign and symptoms include intermittent claudication, discoloration of the involved limb, decreased local temperature, rest pain, ulceration and, bridded nail, loss of subcutaneous fat ,gangrene, absence/feeble distal pulses and recurrent superficial thrombophlebitis ${ }^{1}$. Modern management of this disease includes conservative with use of vasodilators, pentoxyfylline, dose aspirin or platelet aggregation inhibitor drug. Surgical management includes chemical sympathectomy lumbar sympathectomy, and if gangrenous stage is there then amputation is the choice. These means of treatment are costly, not satisfying and associated with complications. In Ayurveda, the disease is not mentioned as it is but the features can be correlated with Gambheera Vatarakta. ${ }^{2}$ Hence, because of Rakta Dusti ,Manjisthadi Kshara Basti ${ }^{3}$ was planned as Ekal Chikitsa. The hypothesis is that, it helps to improve the collateral formation and increased the micro circulation. All these means gave satisfactory results and the patient was able to do his daily activities without any trouble.

Keyword: T.A.O., Thrombo Angiitis Oblitrerans, gambheera vatarakta, manjisthadi Kshara basti 


\section{Introduction:}

In this case the cause of the disease was caused by excessive smoking, the smoking index was $>300$, which is at the higher risk to get the disease. As smoke contains carbon monoxide particles and nicotinic acid they combine with the blood and form carboxyhaemoglobin. Initially it causes vasospasm and hyperplasia of the intima later thrombosis in the vessels and thus obliteration to the blood flow ${ }^{4}$. Clinical features include clauducation, ischaemic changes of the limb, discoloration, ulceration and gangrene. The use of vasodilators, and lumbar sympathectomy, aspirin etc may hold the progression of the disease for a while but is not the permanent solution. Surgical procedures are not having that promising results, if gangrene sets then amputation is the choice but loss of limb is not the choice for the patient.

In Ayurveda no specific correlation can be made with the diseases mentioned in the literature. But, considering the features of the T.A.O it can be said that it is vitiation of blood in the vessels and thus strotodushti especial Raktavah Strotas is present. According to this concept the treatment is planned to remove the sanga i.e obliteration. These two things hold the pathology of the disease related to ayurveda. Patient was treated with ekal chikitsa that is Manjisthadi Ksharabasti in form of yoga basti. The results of this regime gave significantly relief to the patient without any complications and was cost effective.

\section{Case study}

A 48 Years Male Patient
Occupation- Porter

OPD No-143326

IPD No -031020

DOA- 11/9/2017

DOD-

$15 / 10 / 2017$

Address - Hassan

Chief complaints -

- Pain in calf muscle with burning since 4 month

- Discoloration of skin - since 4 month

- Intermittent claudication - since 4 month

- Non healing ulcer over right great toe - since 4 months

- Loss of subcutaneous fat- since 4 months

- Bradded nails- 6 month

History of the patient-The patient was asymptomatic before 4 month then he gradually developed burning sensation on right calf muscle which progressed and turned into pain of the left leg, which didn't allow him to do his daily work, intermittent claudication lead to painful walking. There was discoloration of the limb and an ulcer developed over the right greater toe which did not healed. patient went to modern hospital for the management ,there right lumbar sympathectomy was done, but even after lumbar sympathectomy ${ }^{5}$ ulcer was not healed, and pain was not subsided . so patient came to surgical OPD of sri dhamasthala manjunatheswara ayurveda hospital.

Past history- No history of any major illness 
No history of DM/HTN/Asthma

Surgical History-right Lumbar

Sympathectomy 4 month before

History of drug allergy- patient was not known allergic to any drug or substance.

- Personal history-Diet-mixed

- Appetite-good

- Bowel- normal

- Micturition- normal

- Sleep- disturbed due to pain

- Occupation- driver

- Addiction-chronic smoking

(10 beedis per day from 2 years)

- Tobacco chewing- Yes

- Family history - no specific

- Maternal history- no specific

- Paternal history- no specific

- Self history - no specific

- General examinations-G/C- good

- Temperature-98 F

- Pulse-80/ min

- $\mathrm{BP}-110 / 80 \mathrm{~mm}$ of $\mathrm{Hg}$

- Pallor-negative

- Icterus- negative

- Clubbing- negative

- Cyanosis- negative

- Lymphadenopathy-negative

- Edema- negative

- Systemic examination

- CVS - S1 S2 normal, No added sounds

- $\mathrm{CNS}$ - oriented

- $\mathrm{R} \mathrm{S}-\mathrm{NBVS}$

- P/A - soft

- Sign

- Berger angle-45 degree

- Berger test-positive

- Claudication distance=50 meters
- $\mathrm{L} / \mathrm{E}-$

- Discoloration over skin of right leg=positive

- Hair loss=positive

- Decreased local temperature $=$ positive

Peripheral pulsation

\begin{tabular}{|c|c|c|}
\hline $\begin{array}{l}\text { Peripheral } \\
\text { arteries }\end{array}$ & Right & Left \\
\hline Dorsalis pedis & - & +++ \\
\hline $\begin{array}{c}\text { Posterior } \\
\text { tebial artery }\end{array}$ & - & +++ \\
\hline $\begin{array}{c}\text { Popletial } \\
\text { artery }\end{array}$ & - & ++++ \\
\hline Femoral artery & ++++ & ++++ \\
\hline
\end{tabular}

nvestigations- $\mathrm{Hb}-13 \mathrm{gm} \%$

WBC $-4,300$

$\mathrm{RBC}-4,000$ millions/mm3

Bleeding time- $1 \mathrm{~min} 5 \mathrm{sec}$

Clotting time $-5 \min 0 \mathrm{sec}$

Blood sugar level F- 95 mg/dl

PPBS- $110 \mathrm{mg} / \mathrm{dl}$

Urine routine- NAD

Microscopic - NAD

$H b s A g$ - non reactive

HIV- non reactive

USG abd.- pelvis - Within normal limits

Arterial Doppler left lower limb - normal

Right-occlusion on popliteal artery 
Diagnosis - TAO of right lower limb.

\section{Ayurvedic concept:}

T. A. O. is the inflammation of the wall of artery which form throbus. This can be related to strotodushti so the lakshanas, sangaand vata rakta involving the raktavah strotas so majnisthadi kshara basti has been adopted that Kshara having lekhan property and manjistha having rakta suddhi property ${ }^{6}$. Main principle behind the treatment is removal of the thrombus, subsiding the inflammatory changes and improving the collateral circulation.

\section{Management-}

1. Medicines

- manjisthadi Kshara basti

- jatyadi taila dressing bd

- $\quad$ ingredient of manjisthadi Kshara basti

- saindhav=10 gm

- madhu=50 gm

- manjistha kalka-30 gm

- pancha tikta grita=80 gm

- manjisthadi kashaya=250 ml

- gomutra=100 ml

2. Daily dressing:

The chronic ulcer washed with panchavalkal qwata was daily dressed with jatyadi taila BD

3. Exercise-

Buergers exercise was explained and it was strictly performed by the patient 4 to 5 times a day and 10 minutes each.

\section{4. burger position}

The bed of the patient was elevated 6 inches from head side

\section{Results:}

- Pain- subsided in 1stweek

- Claudication distance- was improved -from $50 \mathrm{~m}$ to $100 \mathrm{~m}$ in 8 days

- Discoloration- skin colour started to improve from 15 days

- Mid night pain-patient was having good sleep because of pain reduction

- Ulcer - the size of the ulcer has reduced.

\section{Discussions:}

Manjistha is the drug having property of rakta shuddhi as well having antiinflammatory property. Gomutra having lekhana property which helps in reducing the size of the thrombus. It is also having anti-inflammatory activity and thus helps n the pain management. Secondly it improves the microcirculation which in helps to maintain the collateral circulation. This helps in increase of the local temperature and hair growth over the limb. Improved collateral chain improved the discolouration of the involved limb, the ischaemic changes didn't progress further. Though there was no obvious visible distal pulsation present but the sign and symptoms of ischaemia subsided due to increased micro circulation. This helped for improving intermittent claudication and thus the claudication distance has improved, rest pain was relieved due to improvement in the ischemia of the nerves.

\section{Conclusion:}

Ayurvedic management for T. A. O. is result oriented, also is cost effective and it 
minimizes the chances of surgical interventional procedures.

\section{References:}

1. Sriram Bhatt, 'Srbs Manual Of Surgery', 4thedition, Jaypee Brothers Medical Publishers.2014: 184.

2. Acharya Charak, Charak Samhita - Vidyotini Vyakhya, Edited By Kashinath Pandey, Chaukhambha Bharati Academy, 22ndedition, Vol. I , Varanasi. 1996: 286.
3. Sharangdhar Samhita, Bramhanand Tripathi, Chaukhamba Surbharti

4. S. Das, A Concise Textbook Of Surgery, 9th Edition, Das Publication,Chapter 15 Disease Of Arteries Page 150 2013: 175.

5. S. Das, A Concise Textbook Of Surgery, 9th Edition, Das Publication,Chapter 15 Disease Of Arteries Page 150 2013: 175.

6. Chakradatta, Indradev Tripathi, Chaukhamba Sanskrit Sansthan, Varanasi, 2005, Pp 542, Page Number 455

Cite article:

Ayurvedic management of Thrombo Angiitis Oblitrerans (TAO)

(Buerger's 